\title{
POINTWISE MULTIPLICATION IN THE REALIZED HOMOGENEOUS BESOV AND TRIEBEL-LIZORKIN SPACES
}

\begin{abstract}
For either homogeneous Besov spaces $\dot{B}_{p, q}^{s}\left(\mathbb{R}^{n}\right)$ or homogeneous Triebel-Lizorkin spaces $\dot{F}_{p, q}^{s}\left(\mathbb{R}^{n}\right)$, with the conditions either $s<n / p$, or $s=n / p$ and $q \leq 1$ in the $\dot{B}_{p, q}^{s}$-case, $p \leq 1$ in the $\dot{F}_{p, q}^{s}$-case, we prove some pointwise multiplication assertions in their realized spaces.
\end{abstract}

Key words: homogeneous Besov space, homogeneous TriebelLizorkin space, pointwise multiplication, realization

\section{Mathematical Subject Classification: 46E35}

1. Introduction and the main result. Let $\dot{B}_{p, q}^{s}\left(\mathbb{R}^{n}\right)$ be the homogeneous Besov space and let $\dot{F}_{p, q}^{s}\left(\mathbb{R}^{n}\right)$ be the homogeneous TriebelLizorkin space. These spaces are abbreviated by $B$ and $F$ in the following. We will use the notation $\dot{A}_{p, q}^{s}\left(\mathbb{R}^{n}\right)$ for either $\dot{B}_{p, q}^{s}\left(\mathbb{R}^{n}\right)$ or $\dot{F}_{p, q}^{s}\left(\mathbb{R}^{n}\right)$ when there is no need to distinguish them. Also, as all function spaces occurring below are defined on Euclidean space $\mathbb{R}^{n}$, we omit $\mathbb{R}^{n}$ in notations.

The space $\dot{A}_{p, q}^{s}$ is defined by distributions modulo polynomials. By means of the "realization" we can consider the version of $\dot{A}_{p, q}^{s}$ in the tempered distribution space $\mathcal{S}^{\prime}$. Recall that G. Bourdaud [2] introduced the realization, which is a linear continuous mapping $\sigma: \dot{A}_{p, q}^{s} \rightarrow \mathcal{S}^{\prime}$ such that $\forall f \in \dot{A}_{p, q}^{s}$ the equivalence class of $\sigma(f)$ modulo polynomials is precisely $f$, i.e., $[\sigma(f)]_{\mathcal{P}}=f$, cf., see Definition 3 below $\left([f]_{\mathcal{P}}\right.$ denotes the equivalence class of a tempered distribution $f$ modulo polynomials). We then obtain the so-called realized space of $\dot{A}_{p, q}^{s}$ denoted by $\dot{\widetilde{A}}_{p, q}^{s}$ (i.e., $\dot{\widetilde{B}}_{p, q}^{s}$ in the $B$-case or $\dot{\widetilde{F}}_{p, q}^{s}$ in the $F$-case), which has a fundamental property that is, under some conditions on $n, s, p$ and $q$, all elements in $\dot{\widetilde{A}}_{p, q}^{s}$ vanish at the infinity 
in the weak sense, see Subsection 2.2 below.

Our aim is to study, in certain cases, the pointwise multiplication in $\dot{\widetilde{A}}_{p, q}^{s}$, since now we have the spaces defined in $\mathcal{S}^{\prime}$ and not in distributions modulo polynomials. Moreover, it is important to say that there is not enough literature on this subject, in comparison to the inhomogeneous case. In this direction, we recall that in [10, Theorem 6.2] it has been proved that any bounded function $f$ such that $[f]_{\mathcal{P}} \in \dot{A}_{p, \infty}^{n / p}$ acts by (pointwise) multiplication on $\dot{\widetilde{A}}_{p, q}^{s}$ if $(n / p-n)_{+}<s<n / p$ in the $B$-case and if $(n / \min (p, q)-n)_{+}<s<n / p$ in the $F$-case. So, we will show principally the pointwise multiplication on $\dot{\widetilde{A}}_{p, q}^{s}$ for $s \leq n / p$, by bounded functions $f$ such that $[f]_{\mathcal{P}}$ belong to $\dot{A}_{p_{1}, \infty}^{\mu}$ for some $\mu>0$ and $p_{1}>0$. So, our principal contribution of this paper is the following statement.

Theorem 1. Let $0<p, p_{1}, p_{2}, q \leq \infty\left(p, p_{1}, p_{2}<\infty\right.$ in the $F$-case $)$ be such that $1 / p=1 / p_{1}+1 / p_{2}$. Let $0 \leq s<\mu<\infty$. Let in addition

either: $(n / p-n)_{+}<s<n / p$ and $0<p<\infty$ in the $B$-case,

$$
(n / \min (p, q)-n)_{+}<s<n / p \text { in the } F \text {-case, }
$$

or : $s=n / p, 0<q \leq 1$ in the $B$-case $(0<p \leq 1$ in the $F$-case),

be satisfied. If $f \in L_{\infty}$ and $g \in \dot{\widetilde{A}}_{p, q}^{s}$ are such that $[f]_{\mathcal{P}} \in \dot{A}_{p_{1}, \infty}^{\mu}$ and $[g]_{\mathcal{P}} \in \dot{A}_{p_{2}, q}^{s-\mu}$, then $f g \in \dot{\widetilde{A}}_{p, q}^{s}$. Moreover, there exists a constant $c>0$ such that

$$
\left\|[f g]_{\mathcal{P}}\right\|_{\dot{A}_{p, q}^{s}} \leq c\left(\|f\|_{\infty}\left\|[g]_{\mathcal{P}}\right\|_{\dot{A}_{p, q}^{s}}+\left\|[f]_{\mathcal{P}}\right\|_{\dot{A}_{p_{1}, \infty}^{\mu}}\left\|[g]_{\mathcal{P}}\right\|_{\dot{A}_{p_{2}, q}^{s-\mu}}\right)
$$

holds, for all such functions $f$ and $g$.

In Theorem 1 the case $s=0$ can be obtained by taking $p=\infty$ in (2) with the $B$-case. Also, we can consider the case $\mu:=s$, by taking the space $\dot{B}_{\infty, 1}^{0}$ instead of $\dot{A}_{p_{2}, q}^{s-\mu}$, since $\dot{B}_{\infty, 1}^{0}$ presents the largest space of $\dot{B}_{\infty, q}^{0}$ when $q \leq 1$, see Theorem 2 below for more details. On the other hand, the condition on $g$ guarantees the "good" representative, indeed, if we replace the assumption $g \in \dot{\widetilde{A}}_{p, q}^{s}$ by $[g]_{\mathcal{P}} \in \dot{A}_{p, q}^{s}$, then, it is possible to fall on a wrong choice of representative which yields a contradiction. For instance, if $g$ is any nonzero polynomial on $\mathbb{R}^{n}$, i.e., $\left\|[g]_{\mathcal{P}}\right\|_{\dot{A}_{p, q}^{s}}=$ 
$=\left\|[g]_{\mathcal{P}}\right\|_{\dot{A}_{p_{2}, q}^{s-\mu}}=0$, then the left hand-side of (3) becomes $\left\|[f g]_{\mathcal{P}}\right\|_{\dot{A}_{p, q}^{s}}=0$ for all such functions $f$, but this is an obviously false assertion.

A consequence of Theorem 1 is the following result which concerns the case $\mu=n / p_{1}$ and $s=n / p$, respectively.

Corollary 1. (i) Let $0<q \leq \infty, 0<p \leq p_{1}<\infty$ and $(n / p-n)_{+}<s<$ $<n / p_{1}$ (with $(n / \min (p, q)-n)_{+}<s<n / p_{1}$ in the $F$-case). Then

$$
\left\|[f g]_{\mathcal{P}}\right\|_{\dot{A}_{p, q}^{s}} \leq c\left(\|f\|_{\infty}+\left\|[f]_{\mathcal{P}}\right\|_{\dot{A}_{p_{1}, \infty}^{n / p_{1}}}\right)\left\|[g]_{\mathcal{P}}\right\|_{\dot{A}_{p, q}^{s}}
$$

holds, for all $f \in L_{\infty}$ such that $[f]_{\mathcal{P}} \in \dot{A}_{p_{1}, \infty}^{n / p_{1}}$ and all $g \in \dot{\widetilde{A}}_{p, q}^{s}$.

(ii) Let $0<p \leq \infty$ and $0<q \leq 1$, in the $B$-case. Let $0<q \leq \infty$ and $0<p \leq 1$, in the $F$-case. Then

$$
\left\|[f g]_{\mathcal{P}}\right\|_{\dot{A}_{p, q}^{n / p}} \leq c\left(\|f\|_{\infty}+\left\|[f]_{\mathcal{P}}\right\|_{\dot{A}_{p, q}^{n / p}}\right)\left\|[g]_{\mathcal{P}}\right\|_{\dot{A}_{p, q}^{n / p}}
$$

holds, for all $f \in L_{\infty}$ such that $[f]_{\mathcal{P}} \in \dot{A}_{p, q}^{n / p}$ and all $g \in \dot{\widetilde{A}}_{p, q}^{n / p}$.

Remark 1. The result [10, Theorem 6.2] is now a particular case of Theorem 1, at least for $s<n / p$ we take $p_{1}$ such that $p \leq p_{1}<n / s$ and apply Corollary 1 (i).

For simplicity of the proofs we split them in two parts. First we decompose the product $f g$ in three terms using the Littlewood-Paley's approach, (we refer to, e.g., [12, Chapter 4], [14, 2.8.1]), then we estimate these terms in $\ell_{q}\left(\mathbb{Z} ; L_{p}\left(\mathbb{R}^{n}\right)\right)$ or $L_{p}\left(\mathbb{R}^{n} ; \ell_{q}(\mathbb{Z})\right)$, which will be given as independent assertions (Propositions 7,10 below). Afterwards, we reduce the proofs to an approximation by smooth functions by considering the case of $g$ being smooth enough. This is principally justified by the Fatou property of the $\dot{A}_{p, q}^{s}$ spaces. Additionally, we will deduce some improvements for the pointwise multiplication on inhomogeneous Besov and Triebel-Lizorkin spaces.

The paper is organized as follows. In Section 2 we collect some necessary facts about $\dot{A}_{p, q}^{s}$. Sections 3 and 4 are devoted to the proof and extensions, respectively.

Notation. The symbol $\mathbb{N}$ denotes the set of natural numbers including $0, \mathbb{Z}$ the integers and $\mathbb{R}$ the real numbers. For $s \in \mathbb{R},[s]$ denotes the greatest integer less than or equal to $s$. For $a \in \mathbb{R}$ we put $a_{+}:=\max (0, a)$. The notation $A \hookrightarrow B$ indicates that $A \subseteq B$ and is continuous. For any function 
$f$, the symbol $f(\cdot)$ means that $x \mapsto f(x)$. With $\|\cdot\|_{p}$ we denote the quasinorm of $L_{p}(0<p \leq \infty)$. If $p \in[1,+\infty]$, we denote by $p^{\prime}$ the conjugate exponent, i.e., $p^{\prime}:=p /(p-1)$. For any quasi-normed space $E$, if $0<q \leq \infty$, then $\ell_{q}(\mathbb{Z} ; E)$ is the set of all sequences $\left(a_{j}\right)_{j \in \mathbb{Z}}$ of elements in $E$ such that $\left\|\left(a_{j}\right)_{j \in \mathbb{Z}}\right\|_{\ell_{q}(\mathbb{Z} ; E)}:=\left(\sum_{j \in \mathbb{Z}}\left\|a_{j}\right\|_{E}^{q}\right)^{1 / q}<\infty$; if $E=\mathbb{R}$ or $\mathbb{C}$ we note $\ell_{q}(\mathbb{Z})$. For brevity, we use the notation $\mathcal{E}_{p, q}$ as $\mathcal{E}_{p, q}:=\ell_{q}\left(\mathbb{Z} ; L_{p}\left(\mathbb{R}^{n}\right)\right)$, i.e., $\left\|\left(f_{j}\right)_{j \in \mathbb{Z}}\right\|_{\mathcal{E}_{p, q}}:=\left(\sum_{j \in \mathbb{Z}}\left\|f_{j}\right\|_{p}^{q}\right)^{1 / q}$, in the $B$-case and $\mathcal{E}_{p, q}:=L_{p}\left(\mathbb{R}^{n} ; \ell_{q}(\mathbb{Z})\right)$, i.e., $\left\|\left(f_{j}\right)_{j \in \mathbb{Z}}\right\|_{\mathcal{E}_{p, q}}:=\left\|\left(\sum_{j \in \mathbb{Z}}\left|f_{j}\right|^{q}\right)^{1 / q}\right\|_{p}$, in the $F$-case, with the usual modification if $p=\infty$ or $q=\infty$. We denote by $\mathcal{P}_{\infty}$ the set of all polynomials in $\mathbb{R}^{n}$. We denote by $\mathcal{S}_{\infty}$ the set of all functions $f$ in the Schawrtz space $\mathcal{S}$ such that $\langle f, u\rangle=0\left(\forall u \in \mathcal{P}_{\infty}\right)$. The symbol $\mathcal{S}_{\infty}^{\prime}$ denotes the topological dual of $\mathcal{S}_{\infty}$. The mapping which takes any $[f]_{\mathcal{P}}$ to the restriction of $f$ to $\mathcal{S}_{\infty}$ turns out to be an isomorphism from $\mathcal{S}^{\prime} / \mathcal{P}_{\infty}$ onto $\mathcal{S}_{\infty}^{\prime}$, for this reason, $\mathcal{S}_{\infty}^{\prime}$ is called the space of distributions modulo polynomials. Sometimes, we will use the Hardy-Littlewood maximal function $M g$ of a locally integrable function $g$ defined as $M g(x):=\sup |Q|^{-1} \int_{Q}|g(y)| \mathrm{d} y$ $\left(\forall x \in \mathbb{R}^{n}\right)$, where the supremum is taken with respect to all cubes $Q$ with sides parallel to the axes and containing $x$. Here $|Q|$ means the Lebesgue measure of the cube $Q$. The standard norms in $\mathcal{S}$ are given by $\zeta_{m}(f):=\sup _{|\alpha| \leq m} \sup _{x \in \mathbb{R}^{n}}(1+|x|)^{m}\left|f^{(\alpha)}(x)\right|(m \in \mathbb{N})$. If $f \in L_{1}$, then $\mathcal{F} f(\xi)=\widehat{f}(\xi):=\int_{\mathbb{R}^{n}} f(x) \mathrm{e}^{-\mathrm{i} x \cdot \xi} \mathrm{d} x$ and $\mathcal{F}^{-1} f$ denote the Fourier transform of $f$ and its inverse Fourier transform, respectively. The operators $\mathcal{F}$ and $\mathcal{F}^{-1}$ can be extended to the whole $\mathcal{S}^{\prime}$ in the usual way. Throughout the paper we use the parameters $s, p$, and $q$ as:

$$
s \in \mathbb{R}, \quad 0<p, q \leq \infty \text { and } p<\infty \text { in the } F \text {-case }
$$

unless otherwise stated. Finally, constants $c, c_{1}, \ldots$ are strictly positive and depend only on the fixed parameters, e.g., $n, s, p, q, \ldots$, and probably on auxiliary functions, their values may vary from line to line.

2. Preliminaries. To introduce the homogeneous as well as the inhomogeneous Besov spaces and Triebel-Lizorkin spaces, the Fouriertheoretical approach via the Littlewood-Paley decomposition presents the basic method. This approach has been developed by Bergh and Löfström [1, Peetre [11, Triebel [14, 15], ...

We choose, once and for all, a radial $C^{\infty}$ function $\rho$, such that $0 \leq \rho \leq 1, \rho(\xi)=1$ if $|\xi| \leq 1$ and $\rho(\xi)=0$ if $|\xi| \geq 3 / 2$. We define $\gamma(\xi):=\rho(\xi)-\rho(2 \xi)$ for all $\xi \in \mathbb{R}^{n}$, with a support located in the compact annulus $1 / 2 \leq|\xi| \leq 3 / 2$. Then it holds $\sum_{j \in \mathbb{Z}} \gamma\left(2^{j} \xi\right)=1(\forall \xi \neq 0)$ and 
$\rho\left(2^{-k} \xi\right)+\sum_{j>k} \gamma\left(2^{-j} \xi\right)=1\left(\forall \xi \in \mathbb{R}^{n}, \forall k \in \mathbb{Z}\right)$. We introduce the convolution operators denoted by $S_{j}$ and $Q_{j}(j \in \mathbb{Z})$, and defined by means of the formulas $\widehat{S_{j} f}(\xi):=\rho\left(2^{-j} \xi\right) \widehat{f}(\xi)$ and $\widehat{Q_{j} f}(\xi):=\gamma\left(2^{-j} \xi\right) \widehat{f}(\xi)$, which are defined on $\mathcal{S}^{\prime}$. The operators $Q_{j}$ are also defined on $\mathcal{S}_{\infty}^{\prime}$ since $Q_{j} f=0$ $(\forall j \in \mathbb{Z})$ if and only if $f$ is a polynomial; then in the following we say

if $f \in \mathcal{S}_{\infty}^{\prime}$ we set $Q_{j} f:=Q_{j} f_{1}$ for all $f_{1} \in \mathcal{S}^{\prime}$ such that $\left[f_{1}\right]_{\mathcal{P}}=f$.

All these operators take values in the space of analytical functions of exponential type, indeed, this fact is an immediate consequence of the Paley-Wiener theorem, see, e.g., [13, Theorem 29.2, p. 311]. Then we obtain the Littlewood-Paley decompositions:

(i) For every $f \in \mathcal{S}_{\infty}\left(\mathcal{S}_{\infty}^{\prime}\right.$, respectively), it holds that $f=\sum_{j \in \mathbb{Z}} Q_{j} f$ in $\mathcal{S}_{\infty}\left(\mathcal{S}_{\infty}^{\prime}\right.$, respectively).

(ii) For every $f \in \mathcal{S}\left(\mathcal{S}^{\prime}\right.$, respectively) and every $k \in \mathbb{Z}$, it holds that $f=S_{k} f+\sum_{j>k} Q_{j} f$ in $\mathcal{S}\left(\mathcal{S}^{\prime}\right.$, respectively).

The operators $S_{j}$ and $Q_{j}$ are uniformly bounded in $\mathcal{L}\left(L_{p}\right)$, for any $p \in[1,+\infty]$ (the Young inequality). Also, we have the following statement proved in, e.g., [10, Proposition 2.5]:

Lemma 1. For any $N \in \mathbb{N}$, there exist constants $c_{1}, c_{2}>0$ and a natural number $m$ such that

(i) $\left\|Q_{j} f\right\|_{p} \leq c_{1} 2^{-j N} \zeta_{m}(f)$ for all $f \in \mathcal{S}$ and all $j \in \mathbb{N}$.

(ii) $\left\|Q_{j} f\right\|_{p}+\left\|S_{j} f\right\|_{p} \leq c_{2} 2^{j N} \zeta_{m}(f)$ for all $f \in \mathcal{S}_{\infty}$ and all $j \in \mathbb{Z} \backslash \mathbb{N}$.

\subsection{Homogeneous Besov and Triebel-Lizorkin spaces.}

Definition 1. The homogeneous Besov space $\dot{B}_{p, q}^{s}$ (or, the homogeneous Triebel-Lizorkin space $\left.\dot{F}_{p, q}^{s}\right)$ is the set of $f \in \mathcal{S}_{\infty}^{\prime}$ such that $\|f\|_{\dot{A}_{p, q}^{s}}:=$ $:=\left\|\left(2^{s j} Q_{j} f\right)_{j \in \mathbb{Z}}\right\|_{\mathcal{E}_{p, q}}<\infty$.

For all $f \in \mathcal{S}^{\prime}$, we define

$$
\|f\|_{\dot{A}_{p, q}^{s}}:=\left\|[f]_{\mathcal{P}}\right\|_{\dot{A}_{p, q}^{s}} .
$$

$\dot{A}_{p, q}^{s}$ becomes a quasi-Banach space in relation to this quasi-norm and it has the following properties:

- $\mathcal{S}_{\infty} \hookrightarrow \dot{A}_{p, q}^{s} \hookrightarrow \mathcal{S}_{\infty}^{\prime}$, which can be obtained easily by Lemma 1 and an estimate of the Nikol'skij representation method type.

- There exist constants $c_{1}, c_{2}>0$ such that

$$
c_{1}\|f\|_{\dot{A}_{p, q}^{s}} \leq \lambda^{n / p-s}\|f(\lambda(\cdot))\|_{\dot{A}_{p, q}^{s}} \leq c_{2}\|f\|_{\dot{A}_{p, q}^{s}} \quad\left(\forall f \in \dot{A}_{p, q}^{s}, \forall \lambda>0\right) .
$$


- $\dot{B}_{p, \min (p, q)}^{s} \hookrightarrow \dot{F}_{p, q}^{s} \hookrightarrow \dot{B}_{p, \max (p, q)}^{s}$ and the following embeddings:

Proposition 1. (See [8]) Let $s_{1}, s_{2} \in \mathbb{R}$ and $0<p_{1}<p_{2}<\infty$ be such that $s_{1}-n / p_{1}=s_{2}-n / p_{2}$. Let $0<q, r \leq \infty$. Then $\dot{B}_{p_{1}, q}^{s_{1}} \hookrightarrow \dot{B}_{p_{2}, q}^{s_{2}} \hookrightarrow$ $\dot{B}_{\infty, q}^{s_{2}-n / p_{2}}, \dot{F}_{p_{1}, q}^{s_{1}} \hookrightarrow \dot{B}_{p_{2}, p_{1}}^{s_{2}}$ and $\dot{F}_{p_{1}, q}^{s_{1}} \hookrightarrow \dot{F}_{p_{2}, r}^{s_{2}}$.

We will need an estimate of the Nikol'skij's type, for which the proof is essentially given, in homogeneous or inhomogeneous case, in [4, Proposition 4], 9, Proposition 3.4], [10, Proposition 2.15], [12, p. 59] and [16].

Proposition 2. Let $a, b$ be real numbers such that $0<a<b$. Let $\left(u_{j}\right)_{j \in \mathbb{Z}}$ be a sequence in $\mathcal{S}^{\prime}$ such that

- $\widehat{u_{j}}$ is supported by the compact annulus $a 2^{j} \leq|\xi| \leq b 2^{j}$,

- $A:=\left\|\left(2^{j s} u_{j}\right)_{j \in \mathbb{Z}}\right\|_{\mathcal{E}_{p, q}}<\infty$.

(i) Then the series $\sum_{j \in \mathbb{Z}} u_{j}$ converges in $\mathcal{S}_{\infty}^{\prime}$ to a limit denoted by $u$ and which satisfies $\|u\|_{\dot{A}_{p, q}^{s}} \leq c A$, where $c$ depends only on $n, s, p, q, a$ and $b$.

(ii) If in addition $s>(n / p-n)_{+}\left(s>(n / \min (p, q)-n)_{+}\right.$in the $F$-case $)$, the same conclusion holds for $a=0$.

We will also need the Fatou property of $\dot{A}_{p, q}^{s}$, see, e.g., [4, Proposition 7] and [9, Proposition 3.13].

Proposition 3. Let $f \in \mathcal{S}_{\infty}^{\prime}$. If there exists a bounded sequence $\left(u_{k}\right)_{k \in \mathbb{N}}$ in $\dot{A}_{p, q}^{s}$ such that $\lim _{k \rightarrow \infty} u_{k}=f$ in $\mathcal{S}_{\infty}^{\prime}$, then $f$ belongs to $\dot{A}_{p, q}^{s}$ and $\|f\|_{\dot{A}_{p, q}^{s}} \leq c \liminf _{k \rightarrow \infty}\left\|u_{k}\right\|_{\dot{A}_{p, q}^{s}}$.

The characterization of $B$ and $F$ spaces by the maximal functions is also useful in what follows. To recall this thing we introduce the maximal operators, thus for $f \in \mathcal{S}_{\infty}^{\prime}, a>0$ and $j \in \mathbb{Z}$, we set

$$
Q_{j}^{*, a} f(x):=\sup _{y \in \mathbb{R}^{n}}\left(1+\left|2^{j} y\right|^{a}\right)^{-1}\left|Q_{j} f(x-y)\right| \quad\left(\forall x \in \mathbb{R}^{n}\right) .
$$

Proposition 4. (See e.g., [7, p. 45]) Let $a>n / p$ in the B-case ( $a>$ $>n / \min (p, q)$ in the $F$-case). Then we have an equivalent quasi-norm in $\dot{A}_{p, q}^{s}$ defined by the expression $\|f\|_{\dot{A}_{p, q}^{s}}^{*}:=\left\|\left(2^{s j} Q_{j}^{*, a} f\right)_{j \in \mathbb{Z}}\right\|_{\mathcal{E}_{p, q}}$.

2.2. Realized spaces. We begin by the following definition:

Definition 2. A distribution $f \in \mathcal{S}^{\prime}$ is said to be vanishing at infinity in the weak sense if $\lim _{\lambda \rightarrow 0} f\left(\lambda^{-1}(\cdot)\right)=0$ in $\mathcal{S}^{\prime}$. The set of all such distributions on $\mathbb{R}^{n}$ is denoted by $\widetilde{C}_{0}$. 
Here are some examples: (i) The elements of $L_{p}$ for $1 \leq p<\infty$. (ii) The derivatives in $\mathcal{D}^{\prime}$ of any element of $\widetilde{C}_{0}$. (iii) The derivatives in $\mathcal{D}^{\prime}$ of a bounded function.

We note that $\mathcal{P}_{\infty} \cap \widetilde{C}_{0}=\{0\}$ (it is an easy assertion). Another example will be given by the following assertion proved in, e.g., [3, Proposition 4.4].

Lemma 2. If $f \in L_{\infty}$ and supp $\widehat{f}$ is a compact subset in $\mathbb{R}^{n} \backslash\{0\}$, then $f \in \widetilde{C}_{0}$.

Now, we give the notion of the realization:

Definition 3. Let $E$ be a vector subspace of $\mathcal{S}_{\infty}^{\prime}$ endowed with a quasiseminorm such that $E \hookrightarrow \mathcal{S}_{\infty}^{\prime}$ holds. A realization of $E$ in $\mathcal{S}^{\prime}$ is a continuous linear mapping $\sigma: E \rightarrow \mathcal{S}^{\prime}$ such that $[\sigma(f)]_{\mathcal{P}}=f$ for all $f \in E$. The image set $\sigma(E)$ is called the realized space of $E$.

By the Littlewood - Paley series we have an example of realization. Let either $s<n / p, \quad$ or $s=n / p \quad$ and $\quad 0<q \leq 1$ in the $B$-case $(0<p \leq 1$ in the $F$-case $)$,

then for any $u \in \dot{A}_{p, q}^{s}$ the series $\sum_{j \in \mathbb{Z}} Q_{j} u$ converges in $\mathcal{S}^{\prime}$, and the linear mapping $\sigma(u):=\sum_{j \in \mathbb{Z}} Q_{j} u$ is a realization on $\dot{A}_{p, q}^{s}$ satisfying $[\sigma(u)]_{\mathcal{P}}=u$ in $\mathcal{S}_{\infty}^{\prime}$ and $\sigma(u) \in \widetilde{C}_{0}$; more precisely, the element $\sigma(u)$ is the unique representative of $u$ that belongs to $\mathcal{S}^{\prime}$, see [3, Proposition 4.6] and [10, Theorems 1.2, 4.1, Section 4.2]. So, if we take $v \in \mathcal{S}^{\prime} \cap \widetilde{C}_{0}$ such that $[v]_{\mathcal{P}} \in \dot{A}_{p, q}^{s}$, we deduce that $\sigma\left([v]_{\mathcal{P}}\right)-v \in \mathcal{P}_{\infty} \cap \widetilde{C}_{0}=\{0\}$. Then, without referencing to the Littlewood - Paley decomposition, we define the realized homogeneous space of $\dot{A}_{p, q}^{s}$ :

Definition 4. Assume that (7) holds. The realized homogeneous space of $\dot{A}_{p, q}^{s}$, denoted by $\dot{\widetilde{A}}_{p, q}^{s}$, is the set of all $f \in \widetilde{C}_{0}$ such that $[f]_{\mathcal{P}} \in \dot{A}_{p, q}^{s}$, and endowed with the quasi-norm $\|f\|_{\dot{\widetilde{A}}_{p, q}^{s}}:=\|f\|_{\dot{A}_{p, q}^{s}}$, see (4).

2.3. Inhomogeneous Besov and Triebel-Lizorkin spaces. The definition of inhomogeneous Besov and Triebel-Lizorkin spaces relies upon Littlewood-Paley theory, similarly to the case of homogeneous spaces.

Definition 5. (i) Let $0<p \leq \infty$. The Besov space $B_{p, q}^{s}$ is the set of $f \in \mathcal{S}^{\prime}$ such that $\|f\|_{B_{p, q}^{s}}:=\left\|S_{0} f\right\|_{p}+\left(\sum_{j \geq 1}\left(2^{s j}\left\|Q_{j} f\right\|_{p}\right)^{q}\right)^{1 / q}<\infty$. 
(ii) Let $0<p<\infty$. The Triebel-Lizorkin space $F_{p, q}^{s}$ is the set of $f \in \mathcal{S}^{\prime}$ such that $\|f\|_{F_{p, q}^{s}}:=\left\|S_{0} f\right\|_{p}+\left\|\left(\sum_{j \geq 1}\left(2^{s j}\left|Q_{j} f\right|\right)^{q}\right)^{1 / q}\right\|_{p}<\infty$.

We denote by $A_{p, q}^{s}$ either $B_{p, q}^{s}$ or $F_{p, q}^{s}$, and recall $\mathcal{S} \hookrightarrow A_{p, q}^{s} \hookrightarrow \mathcal{S}^{\prime}$ and the following statement proved in, e.g., [15, p. 98]; other properties can be found in, e.g., [1, 11, 14, 15.

Proposition 5. Assume that $s>(n / p-n)_{+}$. A function $f$ belongs to $A_{p, q}^{s}$ if and only if $f \in L_{p}$ and $[f]_{\mathcal{P}} \in \dot{A}_{p, q}^{s}$. Moreover, $\|f\|_{p}+\|f\|_{\dot{A}_{p, q}^{s}}$ is an equivalent quasi-norm in $A_{p, q}^{s}$.

2.4. Necessary conditions for pointwise multiplication. By the following assertion, where the proof is similar to that given by Franke [6, Proposition 2.5/1] for $A_{p, q}^{s}$, we can formulate some necessary conditions such that $\left\|f_{1} f_{2}\right\|_{\dot{A}_{p, q}^{s}} \leq c\left\|f_{1}\right\|_{\dot{A}_{p_{1}, q_{1}}^{s_{1}}}\left\|f_{2}\right\|_{\dot{A}_{p_{2}, q_{2}}^{s_{2}}}$ for all $f_{1}, f_{2} \in \mathcal{S}_{\infty}$.

Proposition 6. Let $s_{i} \in \mathbb{R}, 0<p_{i}, q_{i} \leq \infty\left(p_{i}<\infty\right.$ in the $F$-case $)$ $(i=1,2)$. Assume that there exists a constant $c>0$ such that, either $\left\|f_{1} f_{2}\right\|_{\dot{A}_{p, q}^{s}} \leq c\left\|f_{1}\right\|_{\dot{B}_{p_{1}, q_{1}}^{s_{1}}}\left\|f_{2}\right\|_{\dot{A}_{p_{2}, q_{2}}^{s_{2}}}$ or $\left\|f_{1} f_{2}\right\|_{\dot{F}_{p, q}^{s}} \leq c\left\|f_{1}\right\|_{\dot{F}_{p_{1}, q_{1}}^{s_{1}}}\left\|f_{2}\right\|_{\dot{F}_{p_{2}, q_{2}}^{s_{2}}}$ for all $f_{1}, f_{2} \in \mathcal{S}_{\infty}$, are satisfied. Then it follows:

(i) either $s_{i}>s$ or $s_{i}=s$ and $q_{i} \leq q$.

(ii) $s_{1}+s_{2} \geq 0$.

3. Proof.

3.1. Decomposition and estimate of the product. We introduce the bilinear maps $T_{s, \nu, \mu}(f, g):=\left\{2^{k s} Q_{k}\left(\left(S_{k+\nu} f\right)\left(Q_{k+\mu} g\right)\right)\right\}_{k \in \mathbb{Z}}$ and $R_{s, \nu, \mu}(f, g):=\left\{2^{k s} \sum_{j \geq k} Q_{k}\left(\left(Q_{j+\nu} f\right)\left(Q_{j+\mu} g\right)\right)\right\}_{k \in \mathbb{Z}}$, defined on $\mathcal{S}^{\prime} \times \mathcal{S}_{\infty}^{\prime}$ and $\mathcal{S}_{\infty}^{\prime} \times \mathcal{S}_{\infty}^{\prime}$, respectively, where $s \in \mathbb{R}$ and $(\nu, \mu) \in\{-3,-2, \ldots, 3\}^{2}$. In the case $\nu=\mu=0$, we set $T_{s}:=T_{s, 0,0}$ and $R_{s}:=R_{s, 0,0}$.

Proposition 7. Let $f \in L_{\infty}$. Let $g \in L_{p}(0<p \leq \infty)$ be a function of class $C^{\infty}$ such that $\widehat{g}$ has a compact support. Then the product $f g$ is correctly defined in $\mathcal{S}^{\prime}$, and for all $k \in \mathbb{Z}$ we can write

$$
\begin{gathered}
Q_{k}(f g)=\sum_{\nu=-2}^{1} Q_{k}\left(\left(S_{k-3} g\right)\left(Q_{k+\nu} f\right)\right)+\sum_{\nu=-2}^{3} Q_{k}\left(\left(S_{k+1} f\right)\left(Q_{k+\nu} g\right)\right)+ \\
+\sum_{\nu=-3} \sum_{j \geq k+2} Q_{k}\left(\left(Q_{j} f\right)\left(Q_{j+\nu} g\right)\right) \quad \text { in } \mathcal{S}_{\infty}^{\prime}
\end{gathered}
$$

Moreover, if in addition $[f g]_{\mathcal{P}} \in \dot{A}_{p, q}^{s}$ then it holds 


$$
\begin{aligned}
\|f g\|_{\dot{A}_{p, q}^{s}} \leq c & \left(\sum_{\nu=-2}^{1}\left\|T_{s,-3, \nu}(g, f)\right\|_{\mathcal{E}_{p, q}}+\right. \\
& \left.+\sum_{\nu=-2}^{3}\left\|T_{s, 1, \nu}(f, g)\right\|_{\mathcal{E}_{p, q}}+\sum_{\nu=-3}^{1}\left\|R_{s, 0, \nu}(f, g)\right\|_{\mathcal{E}_{p, q}}\right) .
\end{aligned}
$$

Proof. Step 1. Let $\varphi \in \mathcal{S}_{\infty}$. By the Hölder inequality $|\langle g, \varphi\rangle| \leq\|g\|_{p}\|\varphi\|_{p^{\prime}}$ if $p \geq 1$. In the case $0<p<1$ we apply the following lemma proved in, e.g., [11, Lemma 1, p. 54] or [14, Remark 1.4.1/4, p. 23]:

Lemma 3. Let $0<p \leq q \leq \infty$. Then it holds $\|f\|_{q} \leq c R^{n / p-n / q}\|f\|_{p}$, for all $R>0$ and all $f$ satisfying supp $\widehat{f} \subseteq\{\xi:|\xi| \leq R\}$.

We have $|\langle g, \varphi\rangle| \leq\|\varphi\|_{\infty}\|g\|_{1} \leq c\|\varphi\|_{\infty}\|g\|_{p}$ where $c$ depends only on $p$ and supp $\widehat{g}$. This gives $g \in \mathcal{S}_{\infty}^{\prime}$. Let now $\varphi \in \mathcal{S}$, then $|\langle f g, \varphi\rangle| \leq$ $\leq\|f\|_{\infty}\|g \varphi\|_{1}$, and $\|g \varphi\|_{1}$ can be bounded similarly as above. Then $f g \in$ $\in \mathcal{S}^{\prime}$.

Step 2: proof of (8) and (9). Since $f, g$ and $f g$ belong to $\mathcal{S}_{\infty}^{\prime}$, then

$$
Q_{k}(f g)=\sum_{j, \ell \in \mathbb{Z}} Q_{k}\left(\left(Q_{j} f\right)\left(Q_{\ell} g\right)\right) \quad(\forall k \in \mathbb{Z}) .
$$

Denote by $I_{\ell, j}$ and $I_{k}$ intervals $\left[\left(2^{\max (j, \ell)}-3 \cdot 2^{\min (j, \ell)}\right)_{+}, 3\left(2^{j}+2^{\ell}\right)\right]$ and $\left[2^{k}, 3 \cdot 2^{k}\right]$, respectively, for all $j, k, \ell \in \mathbb{Z}$. A careful examination of the intersection of the supports of $\gamma\left(2^{-k}(\cdot)\right)$ and $\mathcal{F}\left(\left(Q_{j} f\right)\left(Q_{\ell} g\right)\right)$ allows us to obtain $\gamma\left(2^{-k} \xi\right) \mathcal{F}\left(\left(Q_{j} f\right)\left(Q_{\ell} g\right)\right)(\xi)=0$ in the following four cases:

- if $j \geq k+2, \quad \ell \geq j+2$ then $2^{\ell}-3 \cdot 2^{j}>3 \cdot 2^{k}, \quad\left(I_{k} \cap I_{\ell, j}=\emptyset\right)$, - if $j \geq k+2, \ell \leq j-4$ then $2^{j}-3 \cdot 2^{\ell}>3 \cdot 2^{k}, \quad\left(I_{k} \cap I_{\ell, j}=\emptyset\right)$,

- if $j \leq k+1, \quad \ell \geq k+4$ then $2^{\ell}-3 \cdot 2^{j}>3 \cdot 2^{k}, \quad\left(I_{k} \cap I_{\ell, j}=\emptyset\right)$, - if $\ell \leq k-3, j \leq k-3$ then $3\left(2^{\ell}+2^{j}\right)<2^{k}, \quad\left(I_{k} \cap I_{\ell, j}=\emptyset\right)$. Consequently, in the right-hand side of 10 we have

$$
\begin{aligned}
\sum_{j, \ell \in \mathbb{Z}} \ldots & =\sum_{j \geq k+2} \sum_{j-3 \leq \ell \leq j+1}+\sum_{j \leq k+1} \sum_{k-2 \leq \ell \leq k+3}+\sum_{k-2 \leq j \leq k+1} \sum_{\ell \leq k-3} \ldots=: \\
& =: W_{k}+V_{k}+U_{k} .
\end{aligned}
$$

We introduce $\widetilde{Q}_{k, 1}:=\sum_{\nu=-2}^{1} Q_{k+\nu}, \widetilde{Q}_{k, 2}:=\sum_{\nu=-2}^{3} Q_{k+\nu}$ and $\widetilde{Q}_{j, 3}:=$ $:=\sum_{\nu=-3}^{1} Q_{j+\nu}$ then $Q_{k}(f g)=U_{k}+V_{k}+W_{k}$ where $U_{k}, V_{k}$, and $W_{k}$ are defined as we want in (8), i.e., we have $U_{k}:=Q_{k}\left(\left(S_{k-3} g\right)\left(\widetilde{Q}_{k, 1} f\right)\right), V_{k}:=$ $:=Q_{k}\left(\left(S_{k+1} f\right)\left(\widetilde{Q}_{k, 2} g\right)\right)$ and $W_{k}:=\sum_{j \geq k+2} Q_{k}\left(\left(Q_{j} f\right)\left(\widetilde{Q}_{j, 3} g\right)\right)$. Finally, (9) follows from the definition of $\|\cdot\|_{\dot{A}_{p, q}^{s}}$ and (8). 
Proposition 8. There exists a constant $c>0$ such that the inequality $\left\|T_{s}(f, g)\right\|_{\mathcal{E}_{p, q}} \leq c\|f\|_{\infty}\|g\|_{\dot{A}_{p, q}^{s}}$ holds, for all $f \in L_{\infty}$ and all $g \in \dot{A}_{p, q}^{s}$.

Proof. Let us recall that $T_{s}$ is defined on $\mathcal{S}^{\prime} \times \mathcal{S}_{\infty}^{\prime}$. On the other hand, we have $\sup _{k \in \mathbb{Z}}\left\|S_{k} f\right\|_{\infty} \leq c\|f\|_{\infty}$. Then

$$
\left|Q_{k}\left(\left(S_{k} f\right)\left(Q_{k} g\right)\right)(x)\right| \leq c\|f\|_{\infty} Q_{k}^{*, a} g(x) \int_{\mathbb{R}^{n}}\left(1+|y|^{a}\right)\left|\mathcal{F}^{-1} \gamma(y)\right| \mathrm{d} y,
$$

for all $x \in \mathbb{R}^{n}$ and all $a>0$ (see $(6)$ for the definition of $Q_{k}^{*, a}$ ). Now, to apply Proposition 4 we choose $a>n / p$ in the $B$-case, and $a>n / \min (p, q)$ in the $F$-case. The desired estimate follows.

Proposition 9. (i) Let $-\infty<s<\mu<\infty$. Let $0<p_{1}, p_{2} \leq \infty$ $\left(p_{1}, p_{2}<\infty\right.$ in the $F$-case) be such that $1 / p=1 / p_{1}+1 / p_{2}$. Then there exists a constant $c>0$ such that $\left\|T_{s}(g, f)\right\|_{\mathcal{E}_{p, q}} \leq c\|f\|_{\dot{A}_{p_{1}, \infty}^{\mu}}\|g\|_{\dot{A}_{p_{2}, q}^{s-\mu}}$ for all $f \in \dot{A}_{p_{1}, \infty}^{\mu}$ and all $g \in \mathcal{S}^{\prime}$ with $[g]_{\mathcal{P}} \in \dot{A}_{p_{2}, q}^{s-\mu}$.

(ii) There exists a constant $c>0$ such that, for all $f \in \dot{A}_{p, q}^{s}$ and all $g \in \mathcal{S}^{\prime}$ with $[g]_{\mathcal{P}} \in \dot{B}_{\infty, 1}^{0}$, it holds $\left\|T_{s}(g, f)\right\|_{\mathcal{E}_{p, q}} \leq c\|f\|_{\dot{A}_{p, q}^{s}}\|g\|_{\dot{B}_{\infty, 1}^{0}}$.

Proof. As in 11], we first have

$$
\left\|T_{s}(g, f)\right\|_{\mathcal{E}_{p, q}} \leq c\left\|\left\{2^{k s}\left(Q_{k}^{*, a} f\right) \sum_{j \leq k} Q_{j}^{*, a} g\right\}_{k \in \mathbb{Z}}\right\|_{\mathcal{E}_{p, q}}(\forall a>0) .
$$

Step 1: proof of (i). We will apply both the following elementary estimate

$$
\left(\sum_{j} \eta_{j}\right)^{d} \leq \sum_{j} \eta_{j}^{d}, \quad 0<d \leq 1, \eta_{j} \geq 0
$$

and the following lemma, which can be proved as in, e.g., [16, Lemma 3.8]:

Lemma 4. For all $0<u \leq \infty$ and all $a>1$, there exists a constant $c>0$, such that $\left\|\left(\sum_{\varepsilon(j-k) \leq 0} a^{\varepsilon(j-k)} \eta_{j}\right)_{k \in \mathbb{Z}}\right\|_{\ell_{u}(\mathbb{Z})} \leq c\left\|\left(\eta_{j}\right)_{j \in \mathbb{Z}}\right\|_{\ell_{u}(\mathbb{Z})} \quad(\varepsilon= \pm 1)$ holds, for all $\left(\eta_{j}\right)_{j \in \mathbb{Z}} \in \ell_{u}(\mathbb{Z})$.

We begin with the $B$-case. We choose $a>n / p$ (then $a>n / p_{1}$ and 
$\left.a>n / p_{2}\right)$, put $d:=\min (1, p)$ and apply the Hölder inequality, then

$$
\begin{aligned}
& \left\|T_{s}(g, f)\right\|_{\ell_{q}\left(L_{p}\right)} \leq c_{1}\left\|\left\{2^{k s}\left(\sum_{j \leq k}\left\|\left(Q_{k}^{*, a} f\right)\left(Q_{j}^{*, a} g\right)\right\|_{p}^{d}\right)^{1 / d}\right\}_{k \in \mathbb{Z}}\right\|_{\ell_{q}(\mathbb{Z})} \leq \\
& \leq c_{1}\left\|\left\{2^{k \mu} \cdot 2^{k(s-\mu)}\left\|Q_{k}^{*, a} f\right\|_{p_{1}}\left(\sum_{j \leq k}\left\|Q_{j}^{*, a} g\right\|_{p_{2}}^{d}\right)^{1 / d}\right\}_{k \in \mathbb{Z}}\right\|_{\ell_{q}(\mathbb{Z})} \leq \\
& \leq c_{2}\|f\|_{\dot{B}_{p_{1}, \infty}^{\mu}}\left(\sum_{k \in \mathbb{Z}}\left\{\sum_{j \leq k} 2^{d(k-j)(s-\mu)}\left(2^{j(s-\mu)}\left\|Q_{j}^{*, a} g\right\|_{p_{2}}\right)^{d}\right\}^{q / d}\right)^{1 / q} .
\end{aligned}
$$

Then by Lemma 4 with $u:=q / d$ we obtain the bound $c\|f\|_{\dot{B}_{p_{1}, \infty}^{\mu}}\|g\|_{\dot{B}_{p_{2}, q}^{s-\mu}}$.

We now consider the $F$-case. We apply the Hölder inequality in 12 , then $\left\|T_{s}(g, f)\right\|_{L_{p}\left(\ell_{q}\right)}$ is bounded by

$$
c\left\|\sup _{\ell \in \mathbb{Z}} 2^{\ell \mu} Q_{\ell}^{*, a} f\right\|_{p_{1}}\left\|\left(\sum_{k \in \mathbb{Z}}\left\{\sum_{j \leq k} 2^{(k-j)(s-\mu)}\left(2^{j(s-\mu)} Q_{j}^{*, a} g\right)\right\}^{q}\right)^{1 / q}\right\|_{p_{2}} ;
$$

so, we choose $a>n / \min (p, q)$, which implies $a>n / \min \left(p_{1}, q\right)$ and $a>n / \min \left(p_{2}, q\right)$, then Lemma 4 with $u:=q$ gives the desired bound $c\|f\|_{\dot{F}_{p_{1}, \infty}^{\mu}}\|g\|_{\dot{F}_{p_{2}, q}^{s-\mu}}$.

Step 2: proof of (ii). In 12 we choose $a>n / \min (p, q) \geq n / p$, then $\left\|T_{s} \overline{(g, f) \|_{\mathcal{E}_{p, q}}} \leq c\right\|\left\{2^{k s} Q_{k}^{*, a} f\right\}_{k \in \mathbb{Z}}\left\|_{\mathcal{E}_{p, q}} \sum_{j \in \mathbb{Z}}\right\| Q_{j}^{*, a} g \|_{\infty}$ which is bounded by $c\|f\|_{\dot{A}_{p, q}^{s}}\|g\|_{\dot{B}_{\infty, 1}^{0}}$. The proof is finished.

Proposition 10. Let $\mu \in \mathbb{R}$. Let $0<p_{1}, p_{2}, r \leq \infty\left(p_{1}, p_{2}<\infty\right.$ in the $F$-case) be such that $1 / p=1 / p_{1}+1 / p_{2}$. Assume that $s>n / p-n$ in the $B$-case and $s>n / \min (p, q)-n$ in the $F$-case. Then there exists a constant $c>0$ such that

$$
\left\|R_{s}(f, g)\right\|_{\mathcal{E}_{p, q}} \leq c\|f\|_{\dot{A}_{p_{1}, q}^{\mu}}\|g\|_{\dot{A}_{p_{2}, r}^{s-\mu}} \quad\left(\forall f \in \dot{A}_{p_{1}, q}^{\mu}, \forall g \in \dot{A}_{p_{2}, r}^{s-\mu}\right) .
$$

In the case $p=p_{1}$ and $p_{2}=\infty$, inequality (14) becomes

$$
\left\|R_{s}(f, g)\right\|_{\mathcal{E}_{p, q}} \leq c\|f\|_{\dot{A}_{p, q}^{\mu}}\|g\|_{\dot{B}_{\infty, r}^{s-\mu}} \quad\left(\forall f \in \dot{A}_{p, q}^{\mu}, \forall g \in \dot{B}_{\infty, r}^{s-\mu}\right) .
$$

Proof. We will use the Marschall pointwise inequality.

Lemma 5. (See e.g., [17, Proposition 6.1]) Let $h \in C^{\infty}$ and $\varphi \in \mathcal{D}$ be such that $\widehat{h}$ and $\varphi$ are supported by the balls $|\xi| \leq A R$ and $|\xi| \leq A$, 
respectively, for some $A>0$ and $R \geq 1$. With $0<t \leq 1$, it follows

$$
\left|\left(\mathcal{F}^{-1} \varphi\right) * h(x)\right| \leq c(A R)^{n / t-n}\|\varphi\|_{\dot{B}_{1, t}^{n / t}}\left(M|h|^{t}(x)\right)^{1 / t},
$$

where $c$ does not depend on $\varphi, h, A, R$ and $x$.

Step 1: the B-case. Applying Lemma 5 with $h:=\left(Q_{j} f\right)\left(Q_{j} g\right), \varphi:=$ $\left.:=\overline{\gamma\left(2^{-k}\right.}(\cdot)\right), A:=3 \cdot 2^{k-1}$ and $R:=2^{j-k+1}(j \geq k)$, then, for some $0<t \leq 1$,

$$
\left|Q_{k}\left(\left(Q_{j} f\right)\left(Q_{j} g\right)\right)(x)\right| \leq c 2^{(j-k)(n / t-n)}\left(M\left|\left(Q_{j} f\right)\left(Q_{j} g\right)\right|^{t}(x)\right)^{1 / t},
$$

for all $j \geq k$ and all $x \in \mathbb{R}^{n}$; here we used the homogeneous property of $\dot{B}_{1, t}^{n / t}$, i.e., $\left\|\gamma\left(2^{-k}(\cdot)\right)\right\|_{\dot{B}_{1, t}^{n / t}} \leq c 2^{-k(n / t-n)}\|\gamma\|_{\dot{B}_{1, t}^{n / t}}$ where $c$ depends only on $n$ and $t$, see (5). We set $d:=\min (1, p)$. Choosing $t$ such that $0<t<p$ (recall that $0<t \leq 1$ ), and using both $(13)$ and the Minkowski inequality with respect to $L_{p / d}\left(\mathbb{R}^{n} ; \ell_{1}(\mathbb{Z})\right)$, we see that $\left\|\sum_{j \geq k} Q_{k}\left(\left(Q_{j} f\right)\left(Q_{j} g\right)\right)\right\|_{p}$ is bounded by

$$
\begin{gathered}
c\left(\int_{\mathbb{R}^{n}}\left\{\sum_{j \geq k} 2^{d(j-k)(n / t-n)}\left(M\left|\left(Q_{j} f\right)\left(Q_{j} g\right)\right|^{t}(x)\right)^{d / t}\right\}^{p / d} \mathrm{~d} x\right)^{1 / p} \leq \\
\leq c\left(\sum_{j \geq k} 2^{d(j-k)(n / t-n)}\left\|M\left|\left(Q_{j} f\right)\left(Q_{j} g\right)\right|^{t}\right\|_{p / t}^{d / t}\right)^{1 / d} .
\end{gathered}
$$

Now, as the maximal function $M$ is bounded on $L_{v}$ if $1<v \leq \infty$ (see [5]), we then apply this assertion with $v:=p / t>1$, and obtain $\left\|M\left|\left(Q_{j} f\right)\left(Q_{j} g\right)\right|^{t}\right\|_{p / t} \leq c\left\|\left(Q_{j} f\right)\left(Q_{j} g\right)\right\|_{p}^{t}$ for some $c>0$ independent of $f, g$, and $j$. Therefore, by the Hölder inequality the term $\left\|R_{s}(f, g)\right\|_{\ell_{q}\left(\mathbb{Z} ; L_{p}\right)}$ is bounded by $c\left(\sum_{k \in \mathbb{Z}} 2^{q k s}\left\{\sum_{j \geq k} 2^{d(j-k)(n / t-n)}\left\|Q_{j} f\right\|_{p_{1}}^{d}\left\|Q_{j} g\right\|_{p_{2}}^{d}\right\}^{q / d}\right)^{1 / q}$, which is bounded by

$$
\begin{aligned}
c\left(\sum_{j \in \mathbb{Z}}\right. & \left.\left(2^{j(s-\mu)}\left\|Q_{j} g\right\|_{p_{2}}\right)^{r}\right)^{1 / r} \times \\
& \times\left(\sum_{k \in \mathbb{Z}}\left\{\sum_{j \geq k} 2^{d(j-k)(n / t-n-s)}\left(2^{j \mu}\left\|Q_{j} f\right\|_{p_{1}}\right)^{d}\right\}^{q / d}\right)^{1 / q} .
\end{aligned}
$$

Now we also choose $n / t-n-s<0$, then it suffices to take $t$ such that $n /(n+s)<t<\min (1, p)$ (see the assumption on $s$ ), then Lemma 4 with $u:=q / d$ yields $\left\|R_{s}(f, g)\right\|_{\ell_{q}\left(\mathbb{Z} ; L_{p}\right)} \leq c\|f\|_{\dot{B}_{p_{1}, q}^{\mu}}\|g\|_{\dot{B}_{p_{2}, r}^{s-\mu}}$. 
Step 2: the F-case. By (16), $\left\|R_{s}(f, g)\right\|_{L_{p}\left(\mathbb{R}^{n} ; \ell_{q}(\mathbb{Z})\right)}$ is estimated by

$$
c\left\|\left(\sum_{k \in \mathbb{Z}}\left\{2^{k s} \sum_{j \geq k} 2^{(k-j)(n-n / t)}\left(M\left|\left(Q_{j} f\right)\left(Q_{j} g\right)\right|^{t}\right)^{1 / t}\right\}^{q}\right)^{1 / q}\right\|_{p} .
$$

Since $0<t \leq 1$, then in 17 the term with $\sum_{j \geq k} \ldots$ is bounded by $\left\{\sum_{j \geq k} 2^{(k-j)(n t-n)} M\left|\left(Q_{j} f\right)\left(Q_{j} g\right)\right|^{t}(x)\right\}^{1 / t}$ for all $x \in \mathbb{R}^{n}$ and all $k \in \mathbb{Z}$. By inserting this estimate into (17), choosing

$$
t<\min (p, q)
$$

and using the fact that $\left\|\left(M h_{k}\right)_{k}\right\|_{L_{v}\left(\mathbb{R}^{n} ; \ell_{w}(\mathbb{Z})\right)} \leq c\left\|\left(h_{k}\right)_{k}\right\|_{L_{v}\left(\mathbb{R}^{n} ; \ell_{w}(\mathbb{Z})\right)}$ holds for $1<v<\infty$ and $1<w \leq \infty$ and for some $c>0$ depending on $n, v$ and $w$ (see [16, Theorem 2.2]); and with $v:=p / t, w:=q / t$ and $h_{k}:=2^{k t s} \sum_{j \geq k} 2^{(k-j)(n t-n)}\left|\left(Q_{j} f\right)\left(Q_{j} g\right)\right|^{t}$, then using the Hölder inequality with $1 / p=1 / p_{1}+1 / p_{2}$, the term $\left\|R_{s}(f, g)\right\|_{L_{p}\left(\mathbb{R}^{n} ; \ell_{q}(\mathbb{Z})\right)}$ is bounded by

$$
\begin{aligned}
c_{1}\left\|\left(\sum_{k \in \mathbb{Z}}\left\{2^{k t s} \sum_{j \geq k} 2^{(k-j)(n t-n)}\left|\left(Q_{j} f\right)\left(Q_{j} g\right)\right|^{t}\right\}^{q / t}\right)^{1 / q}\right\|_{p} \leq \\
\leq c_{2}\left\|\left(\sum_{j \in \mathbb{Z}}\left(2^{j(s-\mu)}\left|Q_{j} g\right|\right)^{r}\right)^{1 / r}\right\|_{p_{2}} \times \\
\times\left\|\left(\sum_{k \in \mathbb{Z}}\left\{\sum_{j \geq k} 2^{(k-j)(n t+s t-n)}\left(2^{j \mu}\left|Q_{j} f\right|\right)^{t}\right\}^{q / t}\right)^{1 / q}\right\|_{p_{1}},
\end{aligned}
$$

under the condition $n /(n+s)<t$; the compatibility of this condition with (18) is guaranteed by assumptions on $s, p, q$. Then we choose $t$ as $n /(n+s)<t<\min (p, q, 1)$, and deduce the bound $c\|f\|_{\dot{F}_{p_{1}, q}^{\mu}}\|g\|_{\dot{F}_{p_{2}, r}^{s-\mu}}$. If $p=p_{1}$, i.e., $p_{2}=\infty$, we estimate 19 by

$$
c\|g\|_{\dot{B}_{\infty, r}^{s-\mu}}\left\|\left(\sum_{k \in \mathbb{Z}}\left\{\sum_{j \geq k} 2^{(k-j)(n t+s t-n)}\left(2^{j \mu}\left|Q_{j} f\right|\right)^{t}\right\}^{q / t}\right)^{1 / q}\right\|_{p}
$$

which yields the correct bound $c\|f\|_{\dot{F}_{p, q}^{\mu}}\|g\|_{\dot{B}_{\infty, r}^{s-\mu}}$.

Remark 2. By easy computations, and owing to the translation invariance on $k \in \mathbb{Z}$ when we take the sum over all $k \in \mathbb{Z}$, Propositions 8, 9 and 10 also hold with $T_{s, \nu, \mu}$ and $R_{s, \nu, \mu}$ (where $(\nu, \mu) \in\{-3,-2, \ldots, 3\}^{2}$ ) instead of $T_{s}$ and $R_{s}$, respectively. 


\subsection{Proof of Theorem 1 and Corollary 1 .}

Proof of Theorem 1, We recall that " $\hookrightarrow$ " indicates a continuous embedding, see the section of Notation in Introduction. Let $f \in L_{\infty}$ and $g \in \dot{\widetilde{A}}_{p, q}^{s}$ be such that $[f]_{\mathcal{P}} \in \dot{A}_{p_{1}, \infty}^{\mu}$ and $[g]_{\mathcal{P}} \in \dot{A}_{p_{2}, q}^{s-\mu}$.

Step 1: proof of $f g \in \mathcal{S}^{\prime}$ and $[f g]_{\mathcal{P}} \in \dot{A}_{p, q}^{s}$. We defined a sequence $\left(g_{k} \overline{k_{k \in \mathbb{N}} \text { by }} g_{k}:=\sum_{|j| \leq k} Q_{j} g=S_{k} g-S_{-k-1} g\right.$, which has the following properties:

- $\widehat{g_{k}}$ is supported by the compact annulus $2^{-k-1} \leq|\xi| \leq 3 \cdot 2^{k-1}$,

- $\left[g_{k}\right]_{\mathcal{P}}$ belongs to $\dot{A}_{p, q}^{s} \cap \dot{A}_{p_{2}, q}^{s-\mu}$ (this follows by Proposition 2 with $\left\|g_{k}\right\|_{\dot{A}_{p, q}^{s}} \leq c\|g\|_{\dot{A}_{p, q}^{s}}$ and $\left\|g_{k}\right\|_{\dot{A}_{p_{2}, q}^{s-\mu}} \leq c\|g\|_{\dot{A}_{p_{2}, q}^{s-\mu}}$ for all $k \in \mathbb{N}$,

- $g_{k} \in C^{\infty} \cap L_{p}$; indeed, if we put $d:=\min (p, 1)$, by the embedding $\dot{A}_{p, q}^{s} \hookrightarrow \dot{B}_{p, \infty}^{s}$ (see Subsection 2.1) it follows $\left\|g_{k}\right\|_{p} \leq\left(\sum_{|j| \leq k}\left\|Q_{j} g\right\|_{p}^{d}\right)^{1 / d}$ which is bounded by $\left(\sum_{|j| \leq k} 2^{-s j d}\right)^{1 / d}\|g\|_{\dot{B}_{p, \infty}^{s}} \leq c(k)\|g\|_{\dot{A}_{p, q}^{s}}$.

Using Propositions 7, 8, 9(i), and 10 with functions $f$ and $g_{k}$ (recall that by Proposition 7, $\left.f g_{k} \in \mathcal{S}^{\prime}\right)$, it holds that the two terms $\left\|T_{s}\left(f, g_{k}\right)\right\|_{\mathcal{E}_{p, q}}$ and $\left\|T_{s}\left(g_{k}, f\right)\right\|_{\mathcal{E}_{p, q}}+\left\|R_{s}\left(f, g_{k}\right)\right\|_{\mathcal{E}_{p, q}}$ can be estimated by $c\|f\|_{\infty}\|g\|_{\dot{A}_{p, q}^{s}}$ and $c\|f\|_{\dot{A}_{p_{1}, \infty}^{\mu}}\|g\|_{\dot{A}_{p_{2}, q}^{s-\mu}}$, respectively. Then, owing to Remark 2 we get

$$
\left\|f g_{k}\right\|_{\dot{A}_{p, q}^{s}} \leq c\left(\|f\|_{\infty}\|g\|_{\dot{A}_{p, q}^{s}}+\|f\|_{\dot{A}_{p_{1}, \infty}^{\mu}}\|g\|_{\dot{A}_{p_{2}, q}^{s-\mu}}\right),
$$

where the constant $c>0$ is independent of $f, g$, and $k$. Now, we claim

$$
\lim _{k \rightarrow \infty}\left(g_{k}-g\right) f=0 \quad \text { in } \quad \mathcal{S}^{\prime} \quad \text { and } \quad f g \in \mathcal{S}^{\prime} .
$$

Indeed, we prove (21) with respect to the cases $s<n / p$ and $s=n / p$, separately:

The case $s<n / p$. (Recall that here $0<p<\infty$ in all spaces, see (1P). Since $g-S_{k} g=\sum_{j>k} Q_{j} g$ (see the beginning of Section 2), we have

$$
\left|\left\langle\left(g_{k}-g\right) f, \varphi\right\rangle\right| \leq\|f\|_{\infty}\left(\left\|\left(S_{-k-1} g\right) \varphi\right\|_{1}+\sum_{j>k}\left\|\left(Q_{j} g\right) \varphi\right\|_{1}\right), \forall \varphi \in \mathcal{S}
$$

and we separately estimate each term in the right-hand side of 22 . If $p \geq 1$, then using the Hölder inequality, we obtain

$$
\begin{gathered}
\sum_{j>k}\left\|\left(Q_{j} g\right) \varphi\right\|_{1} \leq\|\varphi\|_{p^{\prime}} \sum_{j>k}\left\|Q_{j} g\right\|_{p} \leq\|\varphi\|_{p^{\prime}}\|g\|_{\dot{B}_{p, \infty}^{s}} \sum_{j>k} 2^{-s j} \leq \\
\leq c 2^{-s k}\|\varphi\|_{p^{\prime}}\|g\|_{\dot{A}_{p, q}^{s}} \quad \text { (recall that } s>0 \text { ); }
\end{gathered}
$$


if $0<p<1$, we use the Bernstein inequality (see Lemma 3) and obtain

$$
\begin{aligned}
& \sum_{j>k}\left\|\left(Q_{j} g\right) \varphi\right\|_{1} \leq\|\varphi\|_{\infty} \sum_{j>k}\left\|Q_{j} g\right\|_{1} \leq c_{1}\|\varphi\|_{\infty} \sum_{j>k} 2^{j(n / p-n)}\left\|Q_{j} g\right\|_{p} \leq \\
\leq & c_{1} \frac{2^{k(n / p-n-s)}}{1-2^{n / p-n-s}}\|\varphi\|_{\infty}\|g\|_{\dot{B}_{p, \infty}^{s}} \leq c_{2} 2^{k(n / p-n-s)}\|\varphi\|_{\infty}\|g\|_{\dot{A}_{p, q}^{s}} ;
\end{aligned}
$$

again, by the Bernstein inequality we get

$$
\begin{aligned}
& \left\|\left(S_{-k-1} g\right) \varphi\right\|_{1} \leq\|\varphi\|_{1} \sum_{j<-k}\left\|Q_{j} g\right\|_{\infty} \leq c_{1}\|\varphi\|_{1} \sum_{j<-k} 2^{j n / p}\left\|Q_{j} g\right\|_{p} \leq \\
\leq & c_{1} \frac{2^{-k(n / p-s)}}{1-2^{-(n / p-s)}}\|\varphi\|_{1}\|g\|_{\dot{B}_{p, \infty}^{s}} \leq c_{2} 2^{-k(n / p-s)}\|\varphi\|_{1}\|g\|_{\dot{A}_{p, q}^{s}} ;
\end{aligned}
$$

by inserting (23) or (24) (for each case) and (25) into 22 , and by taking $k \rightarrow \infty$ we obtain the convergence of the sequence $\left\{\left(g_{k}-g\right) f\right\}_{k \in \mathbb{N}}$ to 0 in $\mathcal{S}^{\prime}$ since the conditions $\left(\frac{n}{p}-n\right)_{+}<s<\frac{n}{p}$ (in the $B$-case) or $\left(\frac{n}{\min (p, q)}-n\right)_{+}<$ $<s<\frac{n}{p}$ (in the $F$-case) are at our disposal; noticing that in the estimate 24 we have $n / p-n-s<0$ in the $F$-case since $\frac{n}{p}-n \leq\left(\frac{n}{\min (p, q)}-n\right)_{+}<s$. Now, for an arbitrary $\varepsilon>0$, there exists a natural number $k_{\varepsilon}$ such that for all $k \geq k_{\varepsilon}$, it holds

$$
|\langle f g, \varphi\rangle| \leq \varepsilon+\left|\left\langle f g_{k}, \varphi\right\rangle\right|<\infty \quad(\forall \varphi \in \mathcal{S}),
$$

and $f g \in \mathcal{S}^{\prime}$.

The case $s=n / p$ and $0<q \leq 1$ in the $B$-case $(0<p \leq 1$ in the $F$-case $)$.

"This case also includes a situation when we consider $B$-spaces

$$
\text { for } p=\infty \text { (and consequently } s=0) \text {." }
$$

First, we have

$$
\left|f g_{k}(x)-f g(x)\right| \leq\|f\|_{\infty} \sum_{|j|>k}\left\|Q_{j} g\right\|_{\infty} \text { a.e. on } \mathbb{R}^{n},(\forall k \in \mathbb{N}) .
$$

By the embedding $\dot{A}_{p, q}^{n / p} \hookrightarrow \dot{B}_{\infty, 1}^{0}$, which can be obtained by the fact that $\dot{B}_{p, q}^{n / p} \hookrightarrow \dot{B}_{\infty, q}^{0} \hookrightarrow \dot{B}_{\infty, 1}^{0}$ if $0<q \leq 1$ (in the $B$-case) and $\dot{F}_{p, q}^{n / p} \hookrightarrow$ $\hookrightarrow \dot{B}_{\infty, p}^{0} \hookrightarrow \dot{B}_{\infty, 1}^{0}$ if $0<p \leq 1$ (in the $F$-case), we have

$$
\sum_{j \in \mathbb{Z}}\left\|Q_{j} g\right\|_{\infty}=\|g\|_{\dot{B}_{\infty, 1}^{0}} \leq c\|g\|_{\dot{A}_{p, q}^{n / p}}
$$


Then the last term of (28) converges to 0 when $k \rightarrow \infty$, and consequently $f g_{k}$ converges to $f g$ pointwise (i.e., $\lim _{k \rightarrow \infty} f g_{k}(x)=f g(x)$ a.e. on $\left.\mathbb{R}^{n}\right)$. Second, $\left\|g_{k}\right\|_{\infty} \leq \sum_{|j| \leq k}\left\|Q_{j} g\right\|_{\infty} \leq\|g\|_{\dot{B}_{\infty, 1}^{0}}$ implies that $\left\|f g_{k}\right\|_{\infty} \leq c\|f\|_{\infty}\|g\|_{\dot{A}_{p, q}^{n / p}}(\forall k \in \mathbb{N})$. Then applying the Lebesgue dominated convergence theorem, we deduce $\lim _{k \rightarrow \infty} f g_{k}=f g$ in $\mathcal{S}^{\prime}$, and (21) follows as in 26 in this case also.

Now the separate treatment of cases $s<n / p$ and $s=n / p$ is complete and the paragraph below concerns both of them. Trivially, we have $\lim _{k \rightarrow \infty}\left\langle f g_{k}+u, \varphi\right\rangle=\langle f g+v, \varphi\rangle$ for all $\varphi \in \mathcal{S}_{\infty}$ and all $(u, v) \in \mathcal{P}_{\infty} \times \mathcal{P}_{\infty}$, then the sequence $\left(\left[f g_{k}\right]_{\mathcal{P}}\right)_{k \in \mathbb{N}}$ converges to $[f g]_{\mathcal{P}}$ in $\mathcal{S}_{\infty}^{\prime}$ too. Hence, we apply the Fatou property of $\dot{A}_{p, q}^{s}$ in 20) (see Proposition 3. Then the inequality (3) holds.

Step 2: proof of $f g \in \widetilde{C}_{0}$.

Substep 2.1: the case $s<n / p$. (Here $0<p<\infty$, see (1)). We use:

Lemma 6. There exists a constant $c>0$ and a natural number $m \in \mathbb{N}$, such that $|\langle u, \varphi\rangle| \leq c \zeta_{m}(\varphi)\|u\|_{\dot{A}_{p, q}^{s}}, \forall \varphi \in \mathcal{S}, \forall u \in \mathcal{S}^{\prime}$ with $[u]_{\mathcal{P}} \in \dot{A}_{p, q}^{s}$.

Proof of Lemma 6. The bilinear map $\langle\cdot, \cdot\rangle$ is separately continuous on $\dot{A}_{p, q}^{s} \times \mathcal{S}$, then, as $A_{p, q}^{s}$ and $\mathcal{S}$ are Fréchet spaces, this map is continuous; cf., [13, Section 34.2, Corollary, p. 354].

We set $f_{\lambda}:=f\left(\lambda^{-1}(\cdot)\right)$ and $g_{\lambda}:=g\left(\lambda^{-1}(\cdot)\right)$ for all $\lambda>0$. By (5) we have $\left\|g_{\lambda}\right\|_{\dot{A}_{p, q}^{s}} \asymp \lambda^{n / p-s}\|g\|_{\dot{A}_{p, q}^{s}},\left\|g_{\lambda}\right\|_{\dot{A}_{p_{2}, q}^{s-\mu}} \asymp \lambda^{n / p_{2}-s+\mu}\|g\|_{\dot{A}_{p_{2}, q}^{s-\mu}}$ and $\left\|f_{\lambda}\right\|_{\dot{A}_{p_{1}, \infty}^{\mu}} \asymp \lambda^{n / p_{1}-\mu}\|f\|_{\dot{A}_{p_{1}, \infty}^{\mu}}$. By applying, both Lemma 6 with $u:=$ $:=f_{\lambda} g_{\lambda}$, and the estimate (3) with $f_{\lambda}$ and $g_{\lambda}$, we get for all $\varphi \in \mathcal{S}$,

$$
\begin{aligned}
& \left|\left\langle f_{\lambda} g_{\lambda}, \varphi\right\rangle\right| \leq c_{1} \zeta_{m}(\varphi)\left(\|f\|_{\infty}\left\|g_{\lambda}\right\|_{\dot{A}_{p, q}^{s}}+\left\|f_{\lambda}\right\|_{\dot{A}_{p_{1}, \infty}^{\mu}}\left\|g_{\lambda}\right\|_{\dot{A}_{p_{2}, q}^{s-\mu}}\right) \leq \\
& \quad \leq c_{2} \lambda^{n / p-s} \zeta_{m}(\varphi)\left(\|f\|_{\infty}\|g\|_{\dot{A}_{p, q}^{s}}+\|f\|_{\dot{A}_{p_{1}, \infty}^{\mu}}\|g\|_{\dot{A}_{p_{2}, q}^{s-\mu}}\right)
\end{aligned}
$$

for some natural numbers $m$ and some positive constants $c_{1}, c_{2}$ independent of $f, g, \varphi, \lambda$, and $m$. Taking $\lambda \rightarrow 0$ into (29), we obtain the result. Substep 2.2: the case $s=n / p$ and $0<q \leq 1$ in the $B$-case $(0<p \leq 1$ in the $F$-case).

"This case also includes a situation when we consider $B$-spaces for $p=\infty$ (and consequently $s=0$ )."

We defined a sequence $\left(u_{k}\right)_{k \in \mathbb{N}}$ by $u_{k}:=\sum_{|j| \leq k} Q_{j}(f g)$, and 
- $\widehat{u_{k}}$ is supported by the compact annulus $2^{-k-1} \leq|\xi| \leq 3 \cdot 2^{k-1}$,

- $\left\|u_{k}\right\|_{\infty} \leq c\|f g\|_{\dot{A}_{p, q}^{n / p}}(\forall k \geq 0)$, this follows by $\dot{A}_{p, q}^{n / p} \hookrightarrow \dot{B}_{\infty, 1}^{0}$.

These yield $u_{k} \in \widetilde{C}_{0}$, cf., Lemma 2, on the one hand. On the other hand $\sum_{|j|>k}\left\|Q_{j}(f g)\right\|_{\infty} \leq\|f g\|_{\dot{B}_{\infty, 1}^{0}} \leq c\|f g\|_{\dot{A}_{p, q}^{n / p}}(\forall k \in \mathbb{N})$ implies that the first term converges to 0 when $k \rightarrow \infty$. Consequently, we obtain $\lim _{k \rightarrow \infty}\left\|u_{k}-f g\right\|_{\infty}=0$. Now, for all $\varphi \in \mathcal{S}$ and all $\lambda>0$, we get

$$
\left|\left\langle f g\left(\lambda^{-1}(\cdot)\right), \varphi\right\rangle\right| \leq\left\|u_{k}-f g\right\|_{\infty}\|\varphi\|_{1}+\left|\left\langle u_{k}\left(\lambda^{-1}(\cdot)\right), \varphi\right\rangle\right|,
$$

and for an arbitrary $\varepsilon>0$, there exists a number $k_{\varepsilon} \in \mathbb{N}$ such that the right-hand side of the last inequality is bounded by $\varepsilon+\left|\left\langle u_{k}\left(\lambda^{-1}(\cdot)\right), \varphi\right\rangle\right|$ for all $k \geq k_{\varepsilon}$. Thus, by taking $\lambda \rightarrow 0$ we have $\lim _{\lambda \rightarrow 0}\left\langle u_{k}\left(\lambda^{-1}(\cdot)\right), \varphi\right\rangle=0$ implies $\lim _{\lambda \rightarrow 0}\left\langle f g\left(\lambda^{-1}(\cdot)\right), \varphi\right\rangle=0$. Hence we obtain a desired result, and the proof of Theorem 1 is complete.

Before we prove Corollary 1 we need to formulate the complement of Theorem 1 when $\mu=s$ as mentioned in the introduction.

Theorem 2. Assume that either (1) or (2) is satisfied. If $f \in L_{\infty}$ and $g \in \dot{\widetilde{A}}_{p, q}^{s}$ are such that $[f]_{\mathcal{P}} \in \dot{A}_{p, q}^{s}$ and $[g]_{\mathcal{P}} \in \dot{B}_{\infty, 1}^{0}$, then $f g \in \dot{\widetilde{A}}_{p, q}^{s}$. Moreover, there exists a constant $c>0$ such that for all such functions $f$ and $g$ it holds $\|f g\|_{\dot{A}_{p, q}^{s}} \leq c\left(\|f\|_{\infty}\|g\|_{\dot{A}_{p, q}^{s}}+\|f\|_{\dot{A}_{p, q}^{s}}\|g\|_{\dot{B}_{\infty, 1}^{0}}\right)$.

Proof. Using Propositions 7, 8, 9(ii) and the inequality 15$)$ with $(\mu=s$ and $r=1$ ) we obtain 200 with $\|g\|_{\dot{B}_{\infty, 1}^{0}}$ instead of $\|g\|_{\dot{A}_{p_{2}, q}^{s-\mu} \text {. }}$ Then by observations (27) and (30), the proof is similar to that of Theorem 1 .

Proof of Corollary 1. The assertion (i) follows by both, (3) with $\mu:=n / p_{1}$ and $\dot{A}_{p, q}^{s} \hookrightarrow \dot{A}_{p_{2}, q}^{s-n / p_{1}}$ where $p_{2}:=\left(1 / p-1 / p_{1}\right)^{-1}$. For (ii) we apply Theorem 2 with $s=n / p$ and $\dot{B}_{p, q}^{n / p} \hookrightarrow \dot{B}_{\infty, 1}^{0}$ (in $B$-case) and $\dot{F}_{p, q}^{n / p} \hookrightarrow \dot{B}_{\infty, p}^{0} \hookrightarrow \dot{B}_{\infty, 1}^{0}$ (in F-case).

4. An extension to inhomogeneous case. The following two corollaries concern the pointwise multiplication in case of the inhomogeneous spaces $A_{p, q}^{s}$. For brevity, if $E \subseteq \mathcal{S}^{\prime}$, we shall write $f \in E \cap \dot{A}_{p, q}^{s}$ if $f \in E$ and $[f]_{\mathcal{P}} \in \dot{A}_{p, q}^{s}$, also $E \subset \dot{A}_{p, q}^{s}$ means that for arbitrary $f \in E$ we have $[f]_{\mathcal{P}} \in \dot{A}_{p, q}^{s}$.

Corollary 2. Let $0<p \leq p_{1}<\infty$. Assume that $(n / p-n)_{+}<s<$ $<n / p_{1}$ in the $B$-case $\left((n / \min (p, q)-n)_{+}<s<n / p_{1}\right.$ in the $F$-case $)$. Let 
$g \in A_{p, q}^{s}$ and let $f \in L_{\infty}$ be such that $[f]_{\mathcal{P}} \in \dot{A}_{p_{1}, \infty}^{n / p_{1}}$. Then $f g \in A_{p, q}^{s}$. Moreover, there exists a constant $c>0$ such that $\|f g\|_{A_{p, q}^{s}} \leq c\left(\|f\|_{\infty}+\right.$ $\left.+\|f\|_{\dot{A}_{p_{1}, \infty}^{n / p_{1}}}\right)\|g\|_{A_{p, q}^{s}}$. We write $\left(L_{\infty} \cap \dot{A}_{p_{1}, \infty}^{n / p_{1}}\right) \cdot A_{p, q}^{s} \hookrightarrow A_{p, q}^{s}$.

Proof. We first prove that $g \in \widetilde{C}_{0}$. Indeed, if $1 \leq p<\infty$, the assertion follows from the embeddings $A_{p, q}^{s} \subset L_{p} \subset \widetilde{C}_{0}$ (see example (i) just after Definition 2); if $0<p<1$ then $s+n-n / p>0$, and again we have $A_{p, q}^{s} \subset A_{1, q}^{s+n-n / p} \subset L_{1} \subset \widetilde{C}_{0}$. We set $p_{2}:=\left(1 / p-1 / p_{1}\right)^{-1}$. Then the assumption $s<n / p_{1} \leq n / p$ yields $g \in \dot{\widetilde{A}}_{p, q}^{s}$, and $\dot{A}_{p, q}^{s} \hookrightarrow \dot{A}_{p_{2}, q}^{s-n / p_{1}}$ implies $[g]_{\mathcal{P}} \in \dot{A}_{p_{2}, q}^{s-n / p_{1}}$. Then there exist $c_{1}, c_{2}>0$ independent of $g$ such that (see Proposition 5)

$$
\|g\|_{\dot{A}_{p_{2}, q}^{s-n / p_{1}}} \leq c_{1}\|g\|_{\dot{A}_{p, q}^{s}} \leq c_{2}\|g\|_{A_{p, q}^{s}} .
$$

Now, by Theorem 1 (with $\mu:=n / p_{1}$ ) (see also Corollary 1) it holds $\|f g\|_{A_{p, q}^{s}} \leq c\left(\|f g\|_{p}+\|f g\|_{\dot{A}_{p, q}^{s}}\right)$ which is bounded by $c\left(\|f\|_{\infty}\|g\|_{p}+\right.$

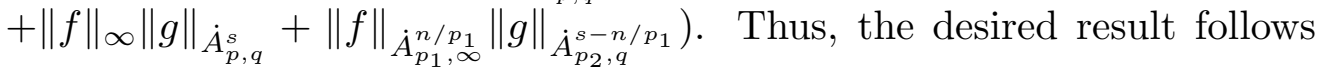
by (31), see again Proposition 5 .

Corollary 3. Let $p, q$ be such that $0<p<\infty$ and $0<q \leq 1$ in the $B$-case, $0<p \leq 1$ and $0<q \leq \infty$ in the F-case. Let $g \in A_{p, q}^{n / p}$ and let $f \in L_{\infty}$ be such that $[f]_{\mathcal{P}} \in \dot{A}_{p, q}^{n / p}$. Then $f g \in A_{p, q}^{n / p}$. Moreover, there exists a constant $c>0$ such that $\|f g\|_{A_{p, q}^{n / p}} \leq c\left(\|f\|_{\infty}+\|f\|_{\dot{A}_{p, q}^{n / p}}\right)\|g\|_{A_{p, q}^{n / p}}$. We write $\left(L_{\infty} \cap \dot{A}_{p, q}^{n / p}\right) \cdot A_{p, q}^{n / p} \hookrightarrow A_{p, q}^{n / p}$.

Proof. Using Proposition 5, Theorem 2, and Corollary 1(ii) the proof is similar to that of Corollary 2, We omit the details.

The main motivation of Corollaries 2 and 3 is that we have now complements of some previous works given in case of $A_{p, q}^{n / p}$. Namely:

Remark 3. (i) Since $A_{p, \infty}^{n / p} \varsubsetneqq \dot{A}_{p_{1}, \infty}^{n / p_{1}}$ if $p_{1} \geq p$, Corollary 2 implies $\left(L_{\infty} \cap A_{p, \infty}^{n / p}\right) \cdot A_{p, q}^{s} \hookrightarrow A_{p, q}^{s}$ if $(n / p-n)_{+}<s<n / p($ with $(n / \min (p, q)-$ $-n)_{+}<s<n / p$ in the $F$-case). If in addition $0<p \leq 1$, we have the result given in [12, Theorem 4.6.2/2(24)-(25), p. 200].

(ii) Because of $A_{p, q_{1}}^{n / p} \hookrightarrow A_{p, q}^{n / p}$ and $A_{p, q_{2}}^{n / p} \varsubsetneqq L_{\infty} \cap \dot{A}_{p, q}^{n / p}$, by Corollary 3 we have the following two assertions: $B_{p, q_{1}}^{n / p} \cdot B_{p, q_{2}}^{n / p} \hookrightarrow B_{p, q}^{n / p}$ if $q:=$ $:=\max \left(q_{1}, q_{2}\right) \leq 1$, and $F_{p, q_{1}}^{n / p} \cdot F_{p, q_{2}}^{n / p} \hookrightarrow B_{p, q}^{n / p}$ if $0<p \leq 1$ and $q:=$ 
$:=\max \left(q_{1}, q_{2}\right)$, given in [12, Theorem 4.6.1/2(20), p. 192] and [12, Theorem 4.6.1/1(8), p. 190], respectively.

Remark 4. The case $p=\infty$ in Corollary 3 can be given as the following:

$$
\left(L_{\infty} \cap \dot{B}_{\infty, q}^{0}\right) \cdot \dot{\widetilde{B}}_{\infty, q}^{0} \hookrightarrow B_{\infty, q}^{0} \quad \text { if } \quad 0<q \leq 1 .
$$

Indeed, assume that $f \in L_{\infty} \cap \dot{B}_{\infty, q}^{0}$ and $g \in \dot{\widetilde{B}}_{\infty, q}^{0}$. By Theorem 2, $f g \in \mathcal{S}^{\prime}$, and as $S_{0}(f g)=\sum_{j \leq 0} Q_{j}(f g)$ then $\left\|S_{0}(f g)\right\|_{\infty} \leq c\|f g\|_{\dot{B}_{\infty, q}^{0}}$. Also, since $\|f g\|_{B_{\infty, q}^{0}} \leq c\left(\left\|S_{0}(f g)\right\|_{\infty}+\|f g\|_{\dot{B}_{\infty, q}^{0}}\right)$, Theorem 2 again yields $\|f g\|_{B_{\infty, q}^{0}} \leq c\left(\|f\|_{\infty}+\|f\|_{\dot{B}_{\infty, q}^{0}}\right)\|g\|_{\dot{B}_{\infty, q}^{0}}$. Now, with respect to

$$
B_{p_{1}, q}^{n / p_{1}} \cdot B_{\infty, q}^{0} \hookrightarrow B_{\infty, q}^{0} \quad \text { if } \quad 0<p_{1}<\infty \quad \text { and } \quad 0<q \leq 1
$$

see [12, Remark 4.4.4/6, p. 180], we have $B_{p_{1}, q}^{n / p_{1}} \varsubsetneqq L_{\infty} \cap \dot{B}_{p_{1}, q}^{n / p_{1}} \subset L_{\infty} \cap$ $\cap \dot{B}_{\infty, q}^{0}$. However, (33) fails in case $p_{1}=\infty$ (see again [12]), then an interesting problem is to obtain 32 with $B_{\infty, q}^{0}$ instead of $L_{\infty} \cap \dot{B}_{\infty, q}^{0}$.

Remark 5. A complement of Remarks 34, that it would be interesting to extend to homogeneous spaces the results given in, e.g., [14], [12, 4.6.14.6.2, pp. 190-207] for inhomogeneous ones.

Acknowledgment. We would like to thank the referees for their valuable comments and suggestions.

\section{References}

[1] Bergh G., Löfström J. Interpolation Theory, An Introduction. Grundlehren Math. Wiss. 223, Springer, Berlin, 1976. DOI: 10.1007/978-3-642-66451-9.

[2] Bourdaud G. Réalisations des espaces de Besov homogènes. Ark. Mat., 1988, vol. 26, pp. 41-54. DOI: 10.1007/BF02386107.

[3] Bourdaud G. Realizations of homogeneous Besov and LizorkinTriebel spaces. Math. Nachr., 2013, vol. 286, pp. 476-491. DOI: 10.1002/mana.201100151.

[4] Bourdaud G., Moussai M., Sickel W. Composition operators in LizorkinTriebel spaces. J. Funct. Anal., 2010, vol. 259, pp. 1098-1128. DOI: 10.1016/j.jfa.2010.04.008.

[5] Fefferman C., Stein E. M. Some maximal inequalities. Amer. J. Math., 1971, vol. 93, pp. 107-115. DOI: 10.2307/2373450. 
[6] Franke J. On the spaces $F_{p, q}^{s}$ of Triebel-Lizorkin type: Pointwise multipliers and spaces on domains. Math. Nachr., 1986, vol. 125, pp. 29-68.

[7] Frazier M., Jawerth B., Weiss G. Littlewood-Paley Theory and the Study of Function Spaces. CBMS-AMS Regional Conference Series, vol. 79, 1991. DOI: $10.1090 / \mathrm{cbms} / 079$.

[8] Jawerth B. Some observations on Besov and Triebel-Lizorkin spaces. Math. Scand., 1977, vol. 40, pp. 94-104. DOI: 10.7146/math.scand.a-11678.

[9] Moussai M. Composition operators on Besov algebras. Rev. Mat. Iberoam., 2012, vol. 28, pp. 239-272. DOI: 10.4171/RMI/676.

[10] Moussai M. Realizations of homogeneous Besov and Triebel-Lizorkin spaces and an application to pointwise multipliers. Anal. Appl. (Singap.), 2015, vol. 13, no. 2, pp. 149-183. DOI: 10.1142/S0219530514500250.

[11] Peetre J. New Thoughts on Besov Spaces. Duke Univ. Math. Series I, Durham, N.C., 1976.

[12] Runst T., Sickel W. Sobolev Spaces of Fractional Order, Nemytskij Operators, and Nonlinear Partial Differential Equations. de Gruyter, Berlin, 1996. DOI: $10.1515 / 9783110812411$

[13] Treves F. Topological Vector Spaces, Distributions and Kernels. Academic Press, INC., 1967.

[14] Triebel H. Theory of Function Spaces. Monogr. Math., vol. 78, Birkhäuser, Basel, 1983. DOI: 10.1007/978-3-0346-0416-1.

[15] Triebel H. Theory of Function Spaces II. Monogr. Math., vol. 84, Birkhäuser, Basel, 1992. DOI: 10.1007/978-3-0346-0419-2.

[16] Yamazaki M. A quasi-homogeneous version of paradifferential operators, I. Boundedness on spaces of Besov type. J. Fac. Sci. Univ. Tokyo, Sect. IA Math., 1986, vol. 33, pp. 131-174.

[17] Yuan W., Sickel W., Yang D. Morrey and Campanato Meet Besov, Lizorkin and Triebel. Springer, vol. 2005, Berlin 2010. DOI: 10.1007/978-3642-14606-0.

Received November 7, $201 \%$.

In revised form, February 9, 2018.

Accepted February 15, 2018.

Published online March 14, 2018.

Laboratory of Functional Analysis and Geometry of Spaces

Mohamed Boudiaf University of M'Sila

28000 M'Sila, Algeria

E-mail: bissarsamira@yahoo.com,mmoussai@yahoo.fr 